\title{
The Influence of Resistance Exercise Training on the Levels of Anxiety in Ischemic Stroke
}

\author{
Felipe José Aidar, ${ }^{1,2}$ Ricardo Jacó de Oliveira, ${ }^{3}$ António José Silva, ${ }^{1}$ Dihogo Gama de Matos, ${ }^{1}$ \\ Mauro Lúcio Mazini Filho, ${ }^{1}$ Robert C. Hickner, ${ }^{4}$ and Victor Machado Reis ${ }^{1}$ \\ ${ }^{1}$ Department of Sports, Science, Exercise, and Health, University of Trás-os-Montes and Alto Douro, 5000-911 Vila Real, Portugal \\ ${ }^{2} 5$ th Battalion of the Military Fire Brigade, Fire Brigade of the State of Minas Gerais, New Horizons Program, \\ 38.406-090 Uberlandia, MG, Brazil \\ ${ }^{3}$ Department of Physical Education, University of Brasília, 70910-900 Brasília, DF, Brazil \\ ${ }^{4}$ Departments of Kinesiology, and Physiology, Center for Health Disparities Research, East Carolina University, \\ Greenville, NC 27858-4353, USA
}

Correspondence should be addressed to Felipe José Aidar, fjaidar@gmail.com

Received 5 May 2012; Revised 24 July 2012; Accepted 17 October 2012

Academic Editor: Disa Sommerfeld

Copyright ( 92012 Felipe José Aidar et al. This is an open access article distributed under the Creative Commons Attribution License, which permits unrestricted use, distribution, and reproduction in any medium, provided the original work is properly cited.

\begin{abstract}
The aim of this pilot study was to analyze the effect of a strength training program on indicators of trait and state anxiety in patients with ischemic stroke. The subjects were divided into two groups: experimental group (EG) consisting of 11 subjects aged $51.7 \pm 8.0$ years and a control group (CG) with 13 subjects aged $52.5 \pm 7.7$ years. EG underwent 12 weeks of strength training, with a frequency of three times a week. For data collection, a State-Trait Anxiety Inventory (STAI) was used. Significant differences were found between pre- and posttest in EG for trait anxiety (43.2 \pm 12.5 pretest $39.9 \pm 7.3$ posttest) and state anxiety (46.9 \pm 7.6 pretest $44.9 \pm 7.7$ posttest) with no differences in CG for trait anxiety ( $42.9 \pm 12.2$ pretest $42.6 \pm 12.1$ posttest) and state anxiety ( $47.4 \pm 8.1$ pretest $47.5 \pm 8.0$ posttest). In the evaluation between the groups, significant differences were found for all indicators of trait anxiety $(39.9 \pm 7.3 \mathrm{EG} ; 42.6 \pm 12.1 \mathrm{CG})$ and state anxiety $(44.9 \pm 7.7 \mathrm{EG} ; 47.5 \pm 8.0 \mathrm{CG})$. This pilot study indicates that strength training may provide an improvement in trait and state anxiety more than one year after stroke.
\end{abstract}

\section{Introduction}

Stroke is linked to cognitive dysfunction and functional impairment, leading to mobility difficulty and interference in social matters that can contribute to depression [1]. Thus, depression and anxiety should be adequately treated to improve functional aspects of life [2]. Anxiety and depression tend to result in decreased adherence to treatment, consequently resulting in reductions in social interactions and motivation. These changes affect overall health [3].

Physical exercises, including strength training, have been used primarily as a form of rehabilitation and may be used for patients who have been affected by stroke. The application of these exercises is a factor which leads to improvements in activities of daily living, posture, and pain reduction [46]. Similarly, strength training has been used as a means of improving cardiopulmonary function in the elderly population, where the occurrence of a stroke is more common [7].

Strength training has currently been listed as a means of promoting improvements in functionality $[8-10]$ and quality of life [11] of individuals who suffered a stroke, especially one of ischemic origin. However, there are few studies evaluating the effects of physical activities, especially those of strength training, in people with sequelae caused by stroke, and their influence on psychosocial aspects. Thus, our aim in this study was to assess the influence of strength training on indicators of trait and state anxiety in people with sequelae of ischemic stroke (IS). 


\section{Methods}

2.1. Sample. The eligibility criteria adopted consisted of subjects having suffered an ischemic stroke at least one year prior to testing, showing hemiplegia or hemiparesis according to Beer et al. [12], having a (loss of motor control on one side of the body, that is, a partial loss of mobility) Duncan [13], and showing no signs of aphasia. Participation in the study was conditional on medical clearance, and only clinically stable subjects were accepted. The present study did not adopt the use of medicines as a criterion for inclusion or exclusion, and the sample description was made using the Rankin Scale [14].

Patient recruitment was done through dissemination of materials through radio, television, and newspaper throughout Minas Gerais that described the program "New Horizons," a project developed by the aforementioned Fire Brigade which supports individuals with special needs at no charge. Twenty nine subjects among those who signed up for the study were consecutively recruited from the 3rd Battalion of the Military Fire Brigade of Belo Horizon, Minas Gerais. Participants were divided at random by lottery into: an experimental group (EG; $N=14$ ) and a control group (CG; $N=15$ ). After the start of the intervention, early in the second week, two subjects left the EG program, leaving EG with 12 subjects. During the remaining study there was one dropout from EG, during treatment and two dropouts from CG for failing to attend the second time point, that is, the posttesting. Thus the EG included 11 individuals, six males and five females, age $51.7 \pm 8.0$ years and the CG included 13 individuals, nine males and four females, age $52.5 \pm 7.7$ years. The subjects underwent a pretest and the EG started strength training after the initial evaluation. The CG continued their normal daily activities and did not receive social or other activity specifically for this study.

The volunteers were informed about the study and signed the authorization according to resolution 196/1996 of the National Health Council, in accordance with the ethical principles contained in the Declaration of Helsinki (1964, revised in 1975, 1983, 1989, 1996, and 2000), and the World Medical Association. The study was approved by the Ethics in Research involving Human Subjects Committee at the University of Trás-os-Montes and Alto Douro, Vila Real, Portugal.

The individuals were classified according to the Rankin Scale (RS) [14]. In the EG, two of the subjects had minor disability (RS 2), seven had moderate disability (RS 3), and two had moderately severe disability (RS 4). As for the CG, two subjects had minor disability, eight had moderate disability, and three had moderately severe disability. There were no asymptomatic subjects, subjects with non-disabling deficit, or subjects with severe disabilities.

\section{Instruments}

The materials used were an indoor basketball court for the warm-up exercises, a Multi Power guided squat apparatus, and a module with eight stations, consisting of horizontal leg press, front pulley, development, bench press, bench press, and advanced. All these equipment were Vênus brand (Vênus, Brazil).

\section{State-Trait Anxiety Inventory (STAI): Form Y}

For the evaluation of the anxiety, a State-Trait Anxiety Inventory (STAI) [15] validated in Brazil under the name IDATE was used [16].

The validation of IDATE has shown good internal consistency [16].

This is a self-assessment questionnaire divided into two parts, one which evaluates trait anxiety, STAI I (referring to personality aspects) and one which evaluates state anxiety, STAI II. Each of these parts consists of 20 statements. Each of the items of both scales has a score 1-4 and the total score can range from 20 (minimum) to 80 (maximum). When answering the questionnaire, the individual must take into consideration that STATE means the way the subject feels at that moment, and trait the way they "usually feel." The scores may indicate low levels of anxiety (00-30), average levels of anxiety (31-49) and high levels of anxiety (greater than or equal to 50); the lower the scores, the lower the levels of anxiety [15]. The cutoff points are used for a classification of the level of anxiety reported by subjects, including special groups, being considered for various types of studies.

\section{The Assessment of Perceived Exertion (Omni Scale)}

The assessment of perceived exertion was performed using the Omni resistance exercise scale [17]. The instructions were given using pictorials to describe the perception of effort during the familiarization and training session. The level of effort was based on a scale ranging from Extremely Easy (0) to Extremely Difficult (10), and the individual should indicate a number corresponding to the exercise intensity [17].

\section{Procedures}

Subjects were submitted to four familiarization sessions before the pretreatment, three for the determination of the load and one for familiarization with the set. The activities were carried out three times a week, with a minimum of 48 hours rest between each session. Each of these sessions lasted 45 to 60 minutes and was conducted between 07:00 and 11:00, according to the availability of the participants. The activities were composed of a warming up session of 10-15 minutes of walking followed by strength training [18]. These subjects performed three sets of $8-10$ repetitions with the same intensity, in accordance with the values of the OMNI Scale. The subjects were also instructed not to hold their breath during the exercises, thus avoiding the Valsalva maneuver and possible subsequent elevation of blood pressure. The adjustment of the loads occurred when the subject reported ease when performing the repetitions. The recovery interval was 120 seconds and was controlled by the subjects with the help of two examiners and an analog clock. EG underwent a strength training program three times 
a week for 12 weeks, and the total average number of sessions for the subjects was 34 training sessions. Shortly after the questionnaires had been filled in, two sessions were carried out for familiarization with the strength exercises as well as with the OMNI scale.

Participants in both groups were asked to report on a posttest questionnaire participation in any kind of purposeful exercise training or physical activity during the course of the study outside of the treatment.

\section{Load Determination}

The 1 repetition maximum (1RM) test is widely recognized as the reference standard for the evaluation of muscular strength [19]. This technique requires an individual to lift the heaviest load possible once through a full range of motion. Although the occurrence of injury may be minimal among experienced weight trainers $[19,20]$, novice exercisers are at greater risk of injury when unaccustomed to handling heavy loads. Additionally, the apprehension of lifting these heavy loads could compromise the performance of inexperienced individuals, causing underestimation of their actual strength.

The 1 RM test was conducted and each subject started the trials with a weight they believed could be lifted once only using maximum effort. Weight increments were then added until they reached the maximum load that could be lifted once. If the participant could not perform a single repetition, 2.4 to $2.5 \%$ were subtracted from the load employed in the test [21]. The subjects rested for 3-5 minutes between the attempts. All subjects underwent three sessions of $1 \mathrm{RM}$ tests with intervals of 48 to 72 hours between each session for the evaluation of muscle strength.

The test was preceded by a warm-up set (10 to 12 repetitions) with approximately $50 \%$ of the load to be used at the first attempt of the $1 \mathrm{RM}$ test. The testing started two minutes after the warm up. The load recorded as 1RM was the one when the individual could complete only one repetition. The transition interval between exercises was 35 minutes. It is noteworthy that the form and the technique used in the performance of each exercise was standardized and continuously monitored in an effort to ensure the quality of the data. In addition, the subjects performed the tests in the same period of the day and did not practice any physical exercise, external to the program, during the experimental period. The 1RM test was conducted before the intervention and after 12 weeks.

\section{Familiarization}

After the individuals had filled in the questionnaires, two familiarization sessions were made with the exercises, as well as with the OMNI scale. During the familiarization, instructions were given on using the scale with the figures mentioned above, which would be used during the intervention. The scale was presented to participants during the strength training sessions, and they were requested to indicate a numerical value on the scale corresponding to their perceived exertion at that moment. The exercises were performed initially with one series, and in a second visit two series were performed until the number of series of the intervention was reached.

\section{Intensity Control}

To ensure safety of the activities, the OMNI scale of perceived exertion, was used according to the procedure adopted by Lagally and Robertson [17]. The scale was presented to participants during the familiarization with strength training, which assigned a numerical value according to their overall perception of effort. The strength training load was adjusted during the intervention in relation to the recommended OMNI scale values of 6-8.

\section{The Administration of the Questionnaires}

The questionnaires were administered before the beginning of the physical activity programs (pretest) and at the end of the 12-week program (posttest). Between the pretest and posttest the EG was subjected to resisted physical activities (strength training), whereas the CG was not subjected to any specific kind of physical activity. This procedure was confirmed by the posttest questionnaire. Questionnaires were administered by the same evaluator who had been used in other studies from our group and had a minimum experience of 20 tests prior to the current study. The evaluator of both pretest and posttest assessments was blinded to patient randomization (EG or CG) throughout the study.

\section{Statistics}

The Wilcoxon signed rank test was used to verify changes within the groups at the end of 12 weeks. Anxiety differences between groups at each evaluation period were tested with the Mann-Whitney $U$ test. A Two-Way ANOVA with a Bonferroni Post Hoc test was used to compare various measurements of strength. Spearman rank correlation was used for the relationship between anxiety and maximum load. The significance level was set to $P<0.05$. The software used for data processing was SPSS version 17.0.

\section{Results}

Before beginning the program, all volunteers said they had spent some time in physical therapy procedures prior to the study, but throughout the program made no other intervention through exercise.

We found significant differences for the experimental group (EG) compared to the control group (CG) in the levels of anxiety, as well as in measures of strength, after strength training (Table 1 and Figure 1). We found significant differences in 1RM strength measures, with EG having more strength than CG after training (Table 1). We found a drop $(P \leq 0.05)$ in anxiety from pre- to posttests for EG, but not for CG (Figure 1). There were also differences $(P \leq 0.05)$ between EG and CG for indicators of state anxiety during the posttest (Figure 1). 
TABLe 1: Maximum load (1RM) in kilograms ( $\mathrm{kg})$ in the pre-and posttest (mean \pm deviation).

\begin{tabular}{lcccc}
\hline & EG pre & EG post & CG pre & CG post \\
\hline Squat & $32.9 \pm 14.2$ & $44.4 \pm 12.4^{*}$ & $33.9 \pm 13.4$ & $34.1 \pm 13.9$ \\
Bench & $19.1 \pm 3.4$ & $36.1 \pm 6.4^{*}$ & $20.1 \pm 4.2$ & $20.3 \pm 4.4$ \\
Leg press & $56.6 \pm 16.5$ & $80.2 \pm 20.4^{*}$ & $54.3 \pm 17.9$ & $54.7 \pm 18.3$ \\
Development & $12.1 \pm 4.3$ & $21.5 \pm 4.8^{*}$ & $13.2 \pm 5.1$ & $12.9 \pm 5.3$ \\
Abdominal & - & - & - & $12.1 \pm 4.5$ \\
Pulley the front & $11.5 \pm 3.9$ & $24.9 \pm 4.1^{*}$ & $30.2 \pm 8.2$ & $11.9 \pm 3.9$ \\
Advanced & $29.5 \pm 6.7$ & $41.9 \pm 6.7^{*}$ & $29.7 \pm 7.3$ \\
\hline
\end{tabular}

${ }^{*} P \leq 0.05$ (two-way ANOVA with Bonferroni post hoc).

—: Not measured

TABLE 2: Correlation between levels of anxiety and maximum load $(1 \mathrm{RM})$ in kilograms $(\mathrm{kg})$ in the experimental group.

\begin{tabular}{lcc}
\hline Exercise & $\begin{array}{c}\text { " } r \text { " spearman } \\
\text { (Trait anxiety) }\end{array}$ & $\begin{array}{c}\text { " } r \text { " spearman } \\
\text { (State anxiety) }\end{array}$ \\
\hline Bar guided squat & -0.827 & -0.717 \\
Machine bech press & -0.834 & -0.814 \\
Horizontal leg press & -0.897 & -0.801 \\
Military press & -0.742 & -0.732 \\
Crunch & - & - \\
Front lat pulldowns & -0.777 & -0.716 \\
Bar guided lunges & -0.819 & -0.779 \\
\hline
\end{tabular}

-: Not measured.

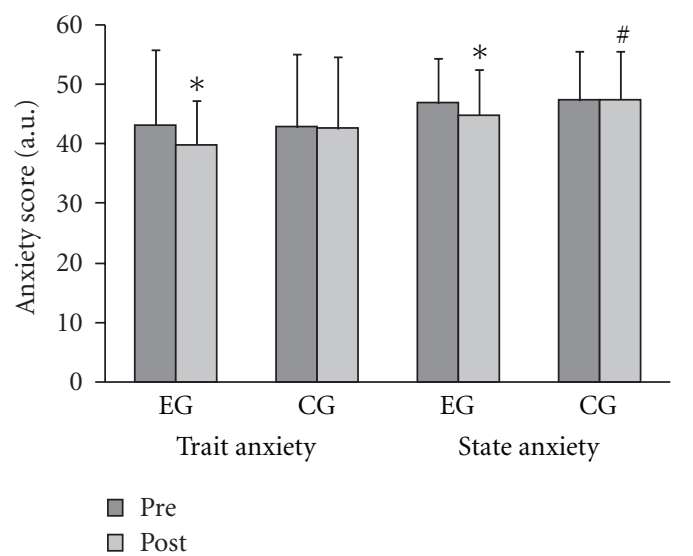

FIGURe 1: Anxiety pre- and poststrength training using the STAI I and II in the experimental (EG, $n=11$ ) and control (CG, $n=$ 13) groups. ${ }^{*} P \leq 0.05$ (Wilcoxon Test). Different from pre in the same group. ${ }^{\#} P \leq 0.05$ (Mann-Whitney Test) Different from the corresponding measure in EG for STATE Anxiety.

We found negative correlations in EG between measures of increased strength gain measured by 1RM and anxiety levels (Table 2), where the highest correlations were with the lower limbs. The negative correlations, measured by a 1RM test, indicated decreases in trait and state anxiety and increase in strength.

\section{Discussion}

The unique findings of this study are that strength training may provide an improvement in trait and state anxiety more than one year after stroke. We found a reduction in both state and trait anxiety in the experimental group in response to a 12-week strength training program. Likewise, when 101 patients with multiple sclerosis have been studied [22] and the relationship between physical dysfunction and depression and anxiety has been examined, it has been concluded that two years after diagnosis these patients have higher levels of depression and anxiety, and these are associated with dysfunctions related to the pathology. Our results extend that study, in that we showed strength training improves physical measures and reduces anxiety. In addition, prior research has shown that symptoms of depression, anxiety and disability are closely related. Therefore, in a study of moderate physical activity and the association between signs of depression, anxiety, and disability in the elderly, physical activity was found to have a modifying effect on symptoms of anxiety. Physical activity plays a central role in reducing disability, and is an effective means of improving psychosocial indicators $[22,23]$.

Regular exercise tends to improve the quality of life [24] and the capacity for work and leisure. Furthermore, regular exercise reduces the probability of new strokes, thereby preventing the resulting decrease in functional ability, stress and anxiety that a new attack would cause [25]. There is evidence that participation in physical activities is the main way to reduce stress in people with disabilities and that it results in improved social functioning and emotional status, leading to reduced anxiety [26]. Research conducted in Brazil analyzed the incidence of depression and anxiety after stroke, showing that, in the long-term, these psychosocial states are very common in people affected by cerebrovascular events [27]. When trying to identify what caused the continuation and increase in levels of depression and anxiety, it has been noted that these indicators are significantly higher in women. Depression and anxiety are associated with issues of employment, educational level, low social activity, cognitive problems, functional dependence on another person, and even prevalence of other pathologies associated with the cerebrovascular events [27]. Anxiety levels remain high even four months after a stroke [28]. These are similar results to those found in the control group in our study, which 
even after three months showed no improvement in levels of trait and state anxiety. Similarly, the immobilization of patients after stroke tends to increase the negative aspects of the pathology, and also contributes to increased indicators of anxiety and irritability. In a previous study of 71 patients with confirmed history of stroke, lack of movement was shown to be an aggravating factor in terms of anxiety [29]. In this sense, lack of mobility can be a factor leading to higher levels of anxiety. It has also been reported in a study from Norway that the measures of emotional health are usually neglected after a cerebrovascular event, that $20-30 \%$ of patients affected by stroke present with anxiety, that 10 $15 \%$ present with emotional imbalance, and that $50-70 \%$ present with initiative reduction and increased fatigue [28, 29]. In addition, medical treatment tends to be based only on prescription medications, with no evidence of successful medical intervention on these psychosocial measures [30].

However, our study is the first to indicate strength training may be beneficial in reducing trait and state anxiety in patients with hemiplegia due to stroke. In relation to the various cut points in the STAI scale there was no change from one major category to the next on the scale. However, there was an improvement in anxiety, as indicated by a statistically significant difference in STAI score. These significant changes detected were perhaps small, but the duration of the study was short (12 weeks). The current results indicate we can detect small improvements in the level of anxiety in as little as 12 weeks with the current program. It is in fact surprised that we were able to detect changes in trait anxiety, which is assumed to be more stable over time than state anxiety, during this short of an intervention period. Further studies are needed to determine if more clinically significant changes can be achieved with longer treatment periods.

Limitations of the present study relate to the sample size, the lack of intervention in the control group, and the size of improvements on the STAI. The small sample size of 11 participants in the experimental group of the present study may have contributed to some of the negative findings due to low statistical power. The lack of a social activity for the control group that was of equal length and frequency to the strength training group is also a limitation of the study, in that some of the improvement in the experimental group may have been due to social interaction rather than strength training per se. Although it could be argued that the measured improvements in the STAI were small, they were statistically significant. The responses were also uniform, in that there were no clear groups of responders as compared to non-responders.

The results of this pilot study indicate that state and trait anxiety may be improved by strength training in persons with minor to moderately severe disability more than one year after stroke. However, this needs to be confirmed in a large randomized controlled trial.

\section{Practical Applications}

Trait and state anxiety is a condition that is normally presented as a result of ischemic stroke sequelae, and is also a factor which limits the performance of activities of daily living. Thus, strength training may improve levels of anxiety, acting as an important means of minimizing the effects of, and preventing the increase in, anxiety caused by ischemic stroke.

\section{Conflict of Interests}

The authors declare no conflict of interests.

\section{References}

[1] Y.-S. Moon, S.-J. Kim, H.-C. Kim, M.-H. Won, and D.-H. Kim, "Correlates of quality of life after stroke," Journal of the Neurological Sciences, vol. 224, no. 1-2, pp. 37-41, 2004.

[2] M. Kimura, R. G. Robinson, and J. T. Kosier, "Treatment of cognitive impairment after poststroke depression: a doubleblind treatment trial," Stroke, vol. 31, no. 7, pp. 1482-1486, 2000.

[3] D. F. Rabelo and A. L. Néri, "Subjective well being and perceived psychological adjustment among old people affected by stroke: a review," Estudos de Psicologia, vol. 11, no. 2, pp. 169$177,2006$.

[4] G. R. Devereux, J. D. Wiles, and I. L. Swaine, "Reductions in resting blood pressure after 4 weeks of isometric exercise training," European Journal of Applied Physiology, vol. 109, no. 4, pp. 601-606, 2010.

[5] C. J. Murrell, J. D Cotter, K. George et al., "Cardiorespiratory and cerebrovascular responses to head-up tilt II: influence of age, training status and acute exercise," Experimental Gerontology, vol. 241, no. 1, pp. 143-151, 2010.

[6] L. Y. Joo, T. S. Yin, D. Xu et al., "A feasibility study using interactive commercial off-the-shelf computer gaming in upper limb rehabilitation in patients after stroke," Journal of Rehabilitation Medicine, vol. 42, no. 5, pp. 437-441, 2010.

[7] D. I. Lovell, R. Cuneo, and G. C. Gass, "Strength training improves submaximum cardiovascular performance in older men," Journal of Geriatric Physical Therapy, vol. 32, no. 3, pp. 117-124, 2009.

[8] M. M. Ouellette, N. K. LeBrasseur, J. F. Bean et al., "Highintensity resistance training improves muscle strength, selfreported function, and disability in long-term stroke survivors," Stroke, vol. 35, no. 6, pp. 1404-1409, 2004.

[9] A. Weiss, T. Suzuki, J. Bean, and R. A. Fielding, "High intensity strength training improves strength and functional performance after stroke," American Journal of Physical Medicine and Rehabilitation, vol. 79, no. 4, pp. 369-376, 2000.

[10] J. D. Moreland, C. H. Goldsmith, M. P. Huijbregts et al., "Progressive resistance strengthening exercises after stroke: a single-blind randomized controlled trial," Archives of Physical Medicine and Rehabilitation, vol. 84, no. 10, pp. 1433-1440, 2003.

[11] S. Studenski, P. W. Duncan, S. Perera, D. Reker, S. M. Lai, and L. Richards, "Daily functioning and quality of life in a randomized controlled trial of therapeutic exercise for subacute stroke survivors," Stroke, vol. 36, no. 8, pp. 1764-1770, 2005.

[12] R. F. Beer, J. P. A. Dewald, and W. Z. Rymer, "Deficits in the coordination of multijoint arm movements in patients with hemiparesis: evidence for disturbed control of limb dynamics," Experimental Brain Research, vol. 131, no. 3, pp. 305-319, 2000.

[13] P. W. Duncan, "Synthesis of intervention trials to improve motor recovery following stroke," Topics in Stroke Rehabilitation, vol. 3, no. 4, pp. 1-20, 1997. 
[14] R. De Haan, M. Limburg, P. Bossuyt, J. Van der Meulen, and N. Aaronson, "The clinical meaning of Rankin "handicap" grades after stroke," Stroke, vol. 26, no. 11, pp. 2027-2030, 1995.

[15] C. D Spielberger, Manual for the State-Trait Anxiety Inventory (STAI: Form Y), Consulting Psychologists, Palo Alto, Calif, USA, 1983.

[16] A. M. Biaggio and L. Natalicio, Manual of the Trait Anxiety Inventory-State (STAI), Applied Psychology Centre EAPC, Rio de Janeiro, Brazil, 1979.

[17] K. M. Lagally and R. J. Robertson, "Construct validity of the OMNI resistance exercise scale," Journal of Strength and Conditioning Research, vol. 20, no. 2, pp. 252-256, 2006.

[18] T. R. Baechle and B. R. Groves, Weight Training: Steps to Success, Human Kinetics, Champaign, Ill, USA, 1998.

[19] American College of Sports Medicine, ACM'S Guidelines for Exercises Testing and Prescription, Williams \& Wilkins, Baltimore, Md, USA, 5th edition, 1995.

[20] T. R. Baechle and R. W. Earle, Essentials of Strength Training and Conditioning, Human Kinetics, Champaign, Ill, USA, 2000.

[21] S. J. Fleck and W. J. Kraemer, Designing Resistance Training Programs, Human Kinectics, Champaign, Ill, USA, 2004.

[22] F. J. Aidar, A. J. Silva, and V. M. Reis, "Estudio de la calidad de vida en el accidente vascular isquémico y su relación con la actividad física," Revista de Neurología, vol. 45, no. 2, pp. 518522, 2007.

[23] Y. Ding, J. Li, Q. Lai et al., "Motor balance and coordination training enhances functional outcome in rat with transient middle cerebral artery occlusion," Neuroscience, vol. 123, no. 3, pp. 667-674, 2004.

[24] F. J. Carod-Artal, "Post-stroke depression (I, II): its differential diagnosis, complications and treatment," Revista de Neurologia, vol. 42, no. 1, pp. 169-175, 2006.

[25] F. J. Carod-Artal, L. Ferreira Coral, D. S. Trizotto, and C. Menezes Moreira, "Poststroke depression: prevalence and determinants in Brazilian stroke patients," Cerebrovascular Diseases, vol. 28, no. 2, pp. 157-165, 2009.

[26] U. Sagen, T. G. Vik, T. Moum, T. Mørland, A. Finset, and T. Dammen, "Screening for anxiety and depression after stroke: comparison of the hospital anxiety and depression scale and the Montgomery and Asberg depression rating scale," Journal of Psychosomatic Research, vol. 67, no. 1, pp. 325-332, 2009.

[27] T. B. Cumming, J. Collier, A. G. Thrift, and J. Bernhardt, "The effect of very early mobilization after stroke on psychological well-being," Journal of Rehabilitation Medicine, vol. 40, no. 8, pp. 609-614, 2008.

[28] B. Fure, "Depression, anxiety and other emotional symptoms after cerebral stroke," Tidsskrift for den Norske Laegeforening, vol. 127, no. 10, pp. 1387-1389, 2007.

[29] V. S. Conn, "Anxiety outcomes after physical activity interventions: meta-analysis findings," Nursing Research, vol. 59, no. 3, pp. 224-231, 2010.

[30] S. Pak and C. Patten, "Strengthening to promote functional recovery poststroke: an evidence-based review," Topics in Stroke Rehabilitation, vol. 15, no. 3, pp. 177-199, 2008. 


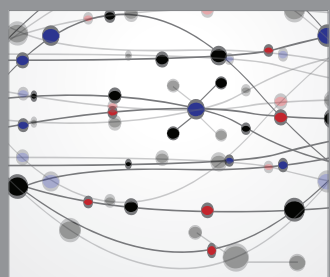

The Scientific World Journal
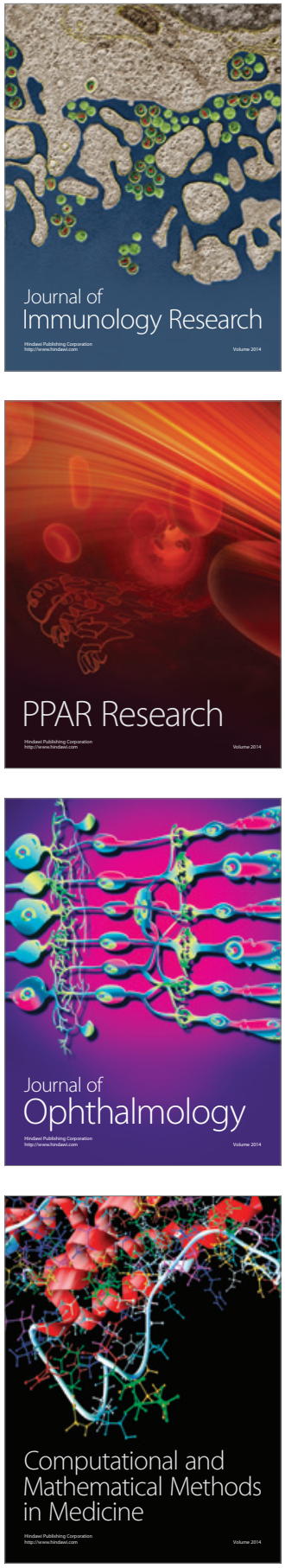

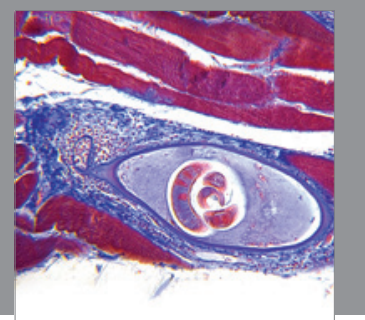

Gastroenterology

Research and Practice
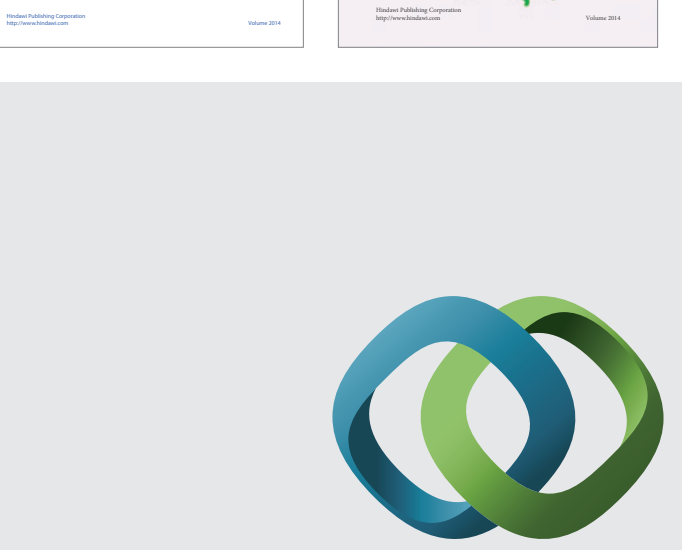

\section{Hindawi}

Submit your manuscripts at

http://www.hindawi.com
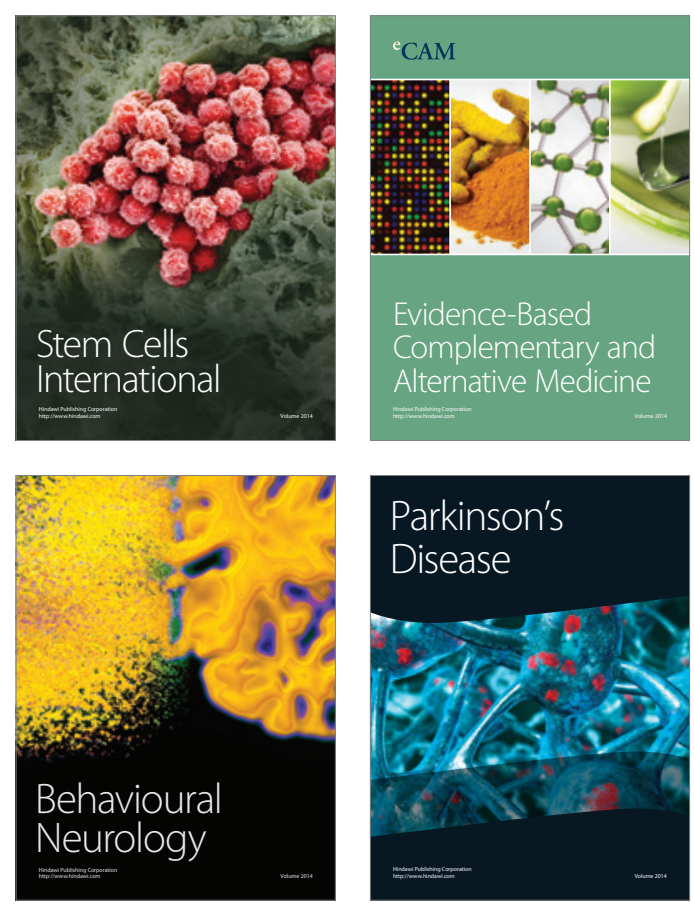

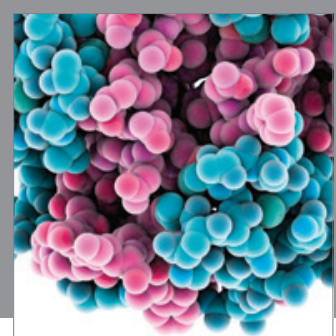

Journal of
Diabetes Research

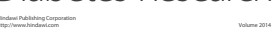

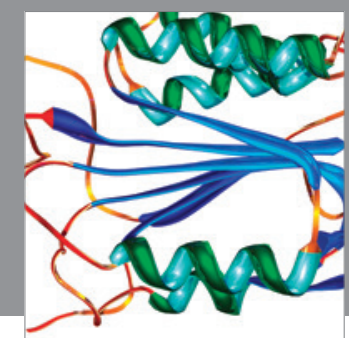

Disease Markers
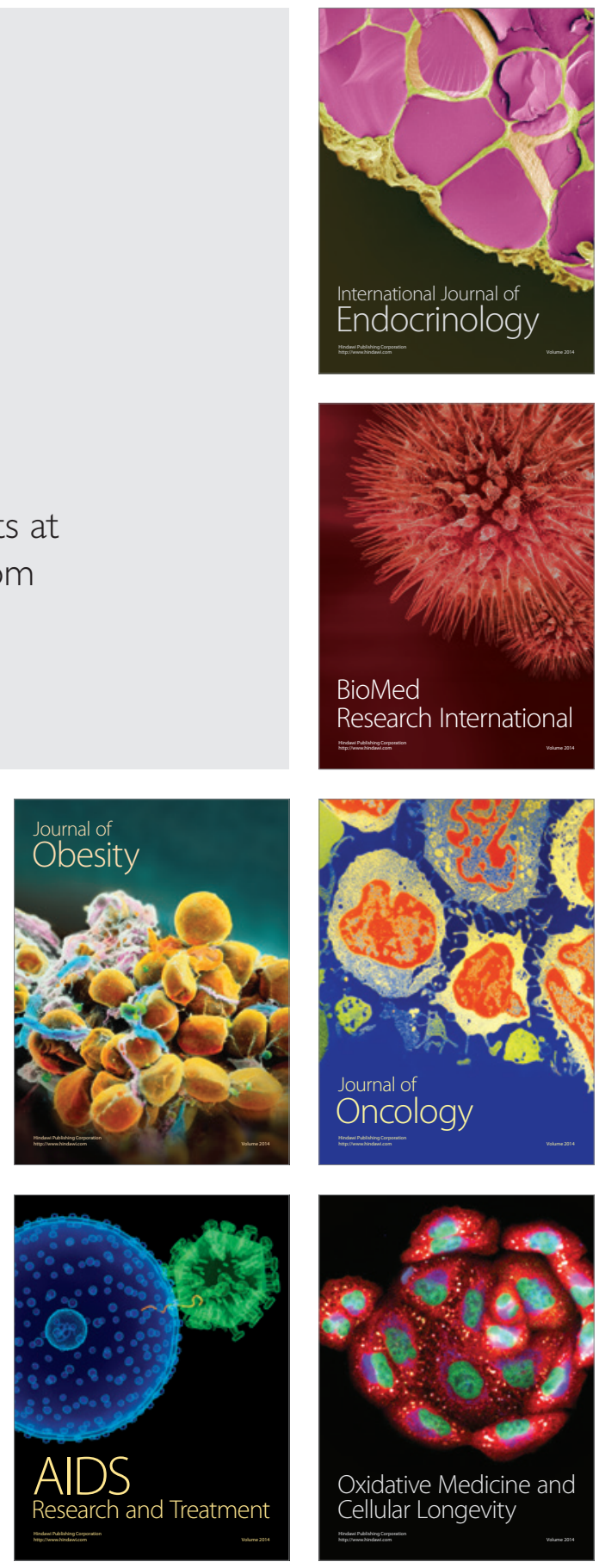